This is a section of Epidemiologia e Psichiatria Sociale, which regularly covers methodological aspects related to the design, conduct, reporting and interpretation of clinical and epidemiological studies. We hope that these editorials will help develop a more critical attitude towards research findings published in the international literature and, additionally, will help promote the implementation of original research projects with higher standards in terms of design, conduct and reporting.

Corrado Barbui, Section Editor and Michele Tansella, Editor EPS

\title{
Why it is important to include unpublished data in systematic reviews
}

\author{
C. Trespidi, C. Barbui and A. Cipriani* \\ Section of Psychiatry and Clinical Psychology, Department of Public Health and Community Medicine, University of Verona, Piazzale L.A. Scuro, \\ 10-37134 Verona, Italy
}

\begin{abstract}
It is known that studies with statistically significant results have a higher probability to be published (publication bias). Therefore, studies with no statistically significant differences (or not favoring the investigational drug) may not be found in commonly accessed databases and remain unpublished. Moreover, unpublished data may also refer to information that are not included in study reports published in scientific journals but that may be important to estimate study outcomes. Retrieving unpublished evidence represents a compelling challenge for researchers, and in the present paper we explore how to do it.
\end{abstract}

Received 9 December 2010; Revised 20 December 2010; Accepted 20 December 2010

Publication bias is 'the tendency on the parts of investigators, reviewers and editors to submit or accept manuscripts for publication based on the direction or strength of the study findings' (Higgins \& Green, 2009). In other words, publication bias means that studies with statistically significant results have a higher probability to be published. Therefore, studies with no statistically significant differences (or not favoring the investigational drug) may not be found in commonly accessed databases, like PubMed or MEDLINE (Cipriani, Girlanda \& Barbui, 2009). Turner and colleagues, who recently analyzed 74 randomized placebo-controlled antidepressant trials submitted to the Food and Drug Administration (FDA) for marketing authorization, found that approximately one-third of all studies went unpublished and that publication status was directly associated with study outcome (Turner et al. 2008). Thirty-seven of 38 studies with positive results were published, whereas only a

* Address for correspondence: Andrea Cipriani, Section of Psychiatry and Clinical Psychology, Department of Public Health and Community Medicine, University of Verona, Piazzale L.A. Scuro, 10-37134 Verona, Italy

(Email: andrea.cipriani@univr.it) minority of studies considered by the FDA as having negative results were published (or were published in a way that conveyed a positive outcome). An inflation of the true estimate of efficacy for antidepressants was shown, with a $32 \%$ increase in the overall effect size of antidepressants in the published literature, as compared with the effect size estimated including unpublished data. Even though in the past some authors have raised some concerns about the reliability of unpublished data as these do not undergo any peer review process (Chalmers et al. 1987), Turner and colleagues clearly highlighted how it is important to have access to unpublished literature when carrying out a systematic review (SR), considering that the main aim of SRs is to provide unbiased estimates of treatment effects (Purgato et al., 2010).

Unpublished data refer to studies that are not published at all but it may also refer to information that are not included in study reports published in scientific journals. As a consequence, retrieving unpublished evidence represents a compelling challenge, and there is no standardized way of collecting it. The first thing to do is to check if there is unpublished material. Nowadays, there are randomized controlled trials' registries where studies have to be registered before 


\begin{tabular}{|c|c|c|}
\hline \multicolumn{3}{|l|}{ Study No.: 29060/356 } \\
\hline \multicolumn{3}{|c|}{$\begin{array}{l}\text { Title: A double-blind, multicentre study to compare paroxetine and fluoxetine in the treatment of patients with } \\
\text { major depressive disorder with regard to antidepressant efficacy, effects on associated anxiety and tolerability. }\end{array}$} \\
\hline \multicolumn{3}{|c|}{ Rationale: This study was designed to compare the efficacy and tolerability of paroxetine and fluoxetine. } \\
\hline \multicolumn{3}{|l|}{ Phase: II-III } \\
\hline \multicolumn{3}{|l|}{ Study Period: 08 Apr $1993-25$ Oct 1994} \\
\hline \multicolumn{3}{|c|}{ Study Design: Multicentre, 8-week, randomised, double blind, comparative study. } \\
\hline \multicolumn{3}{|c|}{ Centres: 10 centres in Australia } \\
\hline \multicolumn{3}{|l|}{ Indication: Major Depressive Disorder (MOD) } \\
\hline \multicolumn{3}{|c|}{ Treatment: Paroxetine $20 \mathrm{mg}$ or fluoxetine $20 \mathrm{mg}$ daily for 8 weeks. } \\
\hline \multicolumn{3}{|c|}{$\begin{array}{l}\text { Primary Outcome/Efficacy Variable: Improvement from baseline in Hamilton Depression Scale (HAM-D) and } \\
\text { change from baseline in Hamilton Anxiety Scale (HAM-A). }\end{array}$} \\
\hline & Paroxetine & Fluoxetine \\
\hline \multicolumn{3}{|l|}{ Number of Subjects: } \\
\hline Planned, $\mathrm{N}$ & 80 & 80 \\
\hline Randomised, $\mathrm{N}$ & 68 & 70 \\
\hline Completed, n (\%) & $47(69)$ & $43(61)$ \\
\hline Total Number Subjects Withdrawn, N (\%) & $21(31)$ & $27(39)$ \\
\hline Withdrawn due to Adverse Events n (\%) & $6(9)$ & $8(11)$ \\
\hline Withdrawn due to Lack of Efficacy $n(\%)^{*}$ & $9(13)$ & $8(11)$ \\
\hline Withdrawn for other reasons $\mathrm{n}(\%)$ & $6(9)$ & $11(16)$ \\
\hline \multicolumn{3}{|l|}{ Primary Efficacy Results: ITT Population } \\
\hline HAM-D(LOCF) & $\begin{array}{l}\text { Paroxetine } \\
\mathrm{N}=64\end{array}$ & $\begin{array}{l}\text { Fluoxetine } \\
\mathrm{N}=68\end{array}$ \\
\hline Baseline, mean (SEM) & $24.6(0.4)$ & $23.8(0.5)$ \\
\hline Day 56, mean (SEM) & $12.0(1.2)$ & $12.6(1.1)$ \\
\hline
\end{tabular}

Fig. 1. Summary of clinical studies in a consistent format, as reported in GlaxoSmithKline (GSK) Clinical Study Register (http:// www.gsk-clinicalstudyregister.com/).

starting recruitment. ClinicalTrials.gov was started in 2000 and now it is the largest single registry of clinical trials (Zarin \& Tse, 2008). This database is freely accessible and in the website some useful information are reported (http://www.clinicaltrial.gov): a summary of the study protocol with participant demographics and baseline characteristics, primary and secondary outcomes and disclosure of agreements between sponsors and researchers; or, for example, it is made clear if the recruitment phase is still ongoing or the study has been completed. A summary of study results is additionally expected to be posted (these data should be available to the public within 12 months of trial completion or within 30 days of FDA approval or clearance of a new drug). Each study has a unique ID number. Trial registration is requested for study approval by the local ethics committees and a policy by the International Committee of Medical Journal Editors requires prospective trial registration as a precondition for publication.

When researchers collect the number of available studies and the corresponding IDs, it is possible to look whether the study has been published or not. If a study has been carried out but not published, full report of study results can be found using internet.

Websites of regulatory agencies are one option, but the most informative sources of unpublished data are pharmaceutical industries' websites. Not all companies have study registries and not all the companies that have study registries report results in an easy-to-use and comprehensive format. In the field of antidepressants, one of the best examples of transparency is the GSK website. As a consequence of a legal settlement between GSK and the New York state following the concerns about the lack of transparency of paroxetine clinical trials in children and adolescents, in 2004 Attorney General's office required GSK to develop a publicly accessible online results database for the timely, comprehensive, posting of results of companymarketed drugs (http://www.gsk-clinicalstudyregister. com/). Fig. 1 shows the format in which study results are presented. Having access to this information may be useful also when the published paper is available because it can help retrieve some missing information (many times standard deviations are not reported in the published tables) or clarify the true number of randomized patients or the exact figures for mean change (it is common that changes in rating scales are reported only on graphs).

In SRs, the inclusion of unpublished data is of utmost relevance, most of all in fields where many studies are available. A pragmatic approach that readers may employ in order to assess if an SR is comprehensive and systematic is to check whether data from unpublished trials are included in the analysis. 


\section{References}

Chalmers TC, Berrier J, Sacks HS, Levin H, Reitman D, Nagalingam P (1987). Meta-analysis of clinical trials as a scientific discipline. I.Replicate variability and comparison of studies that agree and disagree. Statistics in Medicine 6, 733-744.

Cipriani A, Girlanda F, Barbui C (2009). Superiority, equivalence or non-inferiority? Epidemiologia Psichiatria Sociale 18, 311-313.

Higgins JPT, Green S (ed.) (2009). Cochrane Handbook for Systematic Reviews of Interventions 5.0.2 [updated
September 2009]. The Cochrane Collaboration. Available at www.cochrane-handbook.org.

Purgato M, Barbui C, Cipriani A (2010). Assessing risk of bias in randomized controlled trials. Epidemiologia e Psichiatria Sociale 19, 296-297.

Turner EH, Matthews AM, Linardatos E, Tell RA, Rosenthal R (2008). Selective publication of antidepressants trials and its influence on apparent efficacy. New England Journal of Medicine 358, 252-260.

Zarin DA, Tse T (2008). Moving toward transparency of clinical trials. Science 319, 1340-1342. 